\title{
Notes on Mixing Length Theory
}

\author{
Matteo Cantiello ${ }^{1}$ and Yan-Fei Jiang ${ }^{1}$
}

${ }^{1}$ Affiliation not available

December 1, 2021

\section{MLT}

These notes are mostly inspired from reading Cox \& Giuli ("Principles of stellar structure"); some insights are from A.Maeder ("Physics, formation and evolution of rotating stars") and Kippenhahn \& Weigert ("Stellar Structure and Evolution")

\subsection{Pressure Scale Height}

In hydrostatic equilibrium we can define the total pressure scale height, $H_{\mathrm{P}}$, as

$$
-\frac{\mathrm{d} \ln \mathrm{P}}{\mathrm{d} r} \equiv \frac{1}{H_{\mathrm{P}}}=\frac{\rho g}{\mathrm{P}}
$$

where $\mathrm{P}$ is the total pressure $\left(\mathrm{P}_{\text {gas }}+\mathrm{P}_{\text {rad }}\right)$. The pressure scale height is a measure of the distance over which the pressure changes by an appreciable fraction of itself.

\subsection{Gradients}

We can define the gradients $\nabla \equiv \frac{\mathrm{d} \ln T}{\mathrm{~d} \ln \mathrm{P}}$ and $\nabla^{\prime} \equiv \frac{\mathrm{d} \ln T^{\prime}}{\mathrm{d} \ln \mathrm{P}}$. Here $\nabla$ represents the average temperature gradient with respect to pressure of all matter at a given level, while $\nabla^{\prime}$ is the temperature gradient with respect to pressure of a rising/falling fluid element. At first order the temperature excess of such fluid elements can therefore be written as

$$
\Delta T(\Delta r)=T^{\prime}(r+\Delta r)-T(r+\delta r) \simeq \Delta r\left[\frac{\mathrm{d} T^{\prime}}{\mathrm{d} r}-\frac{\mathrm{d} T}{\mathrm{~d} r}\right]
$$

Assuming $T^{\prime} \simeq T$, i.e. the temperature is not changing drastically within the distance $\Delta r$, one can write

$$
\Delta T(\Delta r)=\Delta r T\left[-\frac{\mathrm{d} \ln T}{\mathrm{~d} r}-\left(-\frac{\mathrm{d} \ln T^{\prime}}{\mathrm{d} r}\right)\right]
$$

and using the assumption of pressure equilibrium, the definitions of pressure scale height $H_{\mathrm{P}}$ and the gradients $\nabla$ and $\nabla^{\prime}$ we obtain

$$
\Delta T(\Delta r)=-\frac{\mathrm{d} \ln \mathrm{P}}{\mathrm{d} r} \Delta r T\left[\frac{\mathrm{d} \ln T}{\mathrm{~d} \ln \mathrm{P}}-\left(\frac{\mathrm{d} \ln T^{\prime}}{\mathrm{d} \ln \mathrm{P}}\right)\right]=\Delta r \frac{T}{H_{\mathrm{P}}}\left(\nabla-\nabla^{\prime}\right) .
$$

Note that in general the value of $\nabla^{\prime}$ depends on the rate at which the moving fluid element is exchanging heat with its surroundings. However in the deep interiors of a star a good approximation is $\nabla^{\prime}=\nabla_{\mathrm{ad}} \equiv\left(\frac{\mathrm{d} \ln T}{\mathrm{~d} \ln \mathrm{P}}\right)_{\mathrm{ad}}$, where $\nabla_{\mathrm{ad}}$ is the temperature gradient of a fluid element moving adiabatically. 
Since a non-zero temperature gradient always implies a radiative flux, in a convective region part of the energy is still going to be transported by radiation. This flux can be written as

$$
F_{r}=-\frac{4 a c}{3} \frac{T^{3}}{\kappa \rho} \frac{\mathrm{d} \ln T}{\mathrm{~d} \ln \mathrm{P}}=\frac{4 a c}{3} \frac{T^{4}}{\kappa \rho} \frac{\nabla}{H_{\mathrm{P}}} .
$$

Finally the fictitious (but computable) radiative gradient can be defined as the gradient of temperature required by radiation to carry the total stellar flux:

$$
F=F_{c}+F_{r} \equiv \frac{4 a c}{3} \frac{T^{4}}{\kappa \rho} \frac{\nabla_{r}}{H_{\mathrm{P}}}
$$

In a stellar convection zone where no energy is produced by nuclear reactions, the following inequalities are satisfied: $\nabla_{r}>\nabla>\nabla^{\prime}>\nabla_{\mathrm{ad}}$

\subsection{Convective Flux}

In the "Mixing Length Theory" of convection it is assumed that an element of fluid rises in pressure equilibrium and retains its identity while it moves through a radial distance $\Delta r$, after which it mixes with its surroundings, releasing its excess heat energy. The distance $\Delta r$ is called mixing length and is usually denoted as $\lambda$. The heat transferred by upward moving elements can then be quantified as:

$$
F_{c}=\frac{1}{2} \rho \bar{v} c_{\mathrm{P}} \lambda\left[\frac{\mathrm{d} T^{\prime}}{\mathrm{d} r}-\frac{\mathrm{d} T}{\mathrm{~d} r}\right]
$$

where the factor $1 / 2$ comes from the fact that at each level approximately one-half of the matter is rising and one-half is descending. Here $\rho, \bar{v}$ and $c_{\mathrm{P}}$ are averages along the distance $\lambda$ of density, velocity and specific heat at constant pressure of upward and downward moving fluid elements. Using equation 4 we can write

$$
F_{c}=\frac{1}{2} \rho \bar{v} c_{\mathrm{P}} T \frac{\lambda}{H_{\mathrm{P}}}\left(\nabla-\nabla^{\prime}\right)
$$

\subsection{Convective Velocities}

We can calculate the accelaration of a convective fluid element

$$
\ddot{r}=-g-\frac{1}{\rho} \frac{\partial \mathrm{P}}{\partial r}
$$

which expanded at first order around the equilibrium state leads to an estimate of the total net force (buoyant minus gravitation) $f=-g \Delta \rho$. Along the distance $\Delta r$ a convective fluid element experience the force $f(\Delta r)=-g \Delta \rho(\Delta r)$. Here the variation of $g$ with height is considered negligible. The work done per unit volume over the distance $\Delta r$ is therefore

$$
W(\Delta r)=\int_{0}^{\Delta r} f(x) \mathrm{d} x=-g \int_{0}^{\Delta r} \Delta \rho(x) \mathrm{d} x=-\frac{1}{2} g \Delta \rho(\Delta r) \Delta r .
$$

An average of $W(\Delta r)$ over all possible values of $\Delta r$ and a choice of a numerical factor $(1 / 4)$ in exchanging $\Delta r$ with $\lambda$ leads to the following expression ( $W$ depends quadratically on $\Delta r$ ):

$$
\bar{W}(\lambda)=\frac{1}{4} W(\lambda)=-\frac{1}{8} g \Delta \rho(\lambda) \lambda .
$$

Under the assumption of no dissipative forces, no transfer of kinetic energy to surrounding matter and no heat loss, then one would conclude $\bar{W}(\lambda)=1 / 2 \rho v^{2}$. However in the MLT one assumes that only half of this work is transformed into kinetic energy. This leads to

$$
\overline{\frac{1}{2} \rho v^{2}} \simeq \frac{1}{2} \rho \bar{v}^{2}=\frac{1}{2} \bar{W}(\lambda)=-\frac{1}{16} g \Delta \rho(\lambda) \lambda,
$$


so that the velocity of convective elements at the level where the mixing length is $\lambda$ can be expressed as

$$
\bar{v}^{2}=-\frac{1}{8} g(\Delta \rho(\lambda) / \rho) \lambda
$$

Relating $\Delta \rho$ and $\Delta T$ requires the equation of state for the gas $\rho=\rho(\mu, T, \mathrm{P})$, which in differential form can be written as

$$
\begin{gathered}
\mathrm{d} \rho=\left(\frac{\partial \rho}{\partial \mu}\right)_{\mathrm{P}, T} \mathrm{~d} \mu+\left(\frac{\partial \rho}{\partial T}\right)_{\mathrm{P}, \mu} \mathrm{d} T+\left(\frac{\partial \rho}{\partial \mathrm{P}}\right)_{\mu, T} \mathrm{dP} \\
\mathrm{d} \rho=\frac{\rho}{\mu}\left(\frac{\partial \ln \rho}{\partial \ln \mu}\right)_{\mathrm{P}, T} \mathrm{~d} \mu+\frac{\rho}{T}\left(\frac{\partial \ln \rho}{\partial \ln T}\right)_{\mathrm{P}, \mu} \mathrm{d} T+\frac{\rho}{\mathrm{P}}\left(\frac{\partial \ln \rho}{\partial \ln \mathrm{P}}\right)_{\mu, T} \mathrm{dP}
\end{gathered}
$$

and finally

$$
\frac{\mathrm{d} \rho}{\rho}=\alpha \frac{\mathrm{dP}}{\mathrm{P}}-\delta \frac{\mathrm{d} T}{T}+\phi \frac{\mathrm{d} \mu}{\mu}
$$

Where $\phi \equiv\left(\frac{\partial \ln \rho}{\partial \ln \mu}\right)_{\mathrm{P}, T}, \quad \delta \equiv-\left(\frac{\partial \ln \rho}{\partial \ln T}\right)_{\mathrm{P}, \mu}, \quad \alpha \equiv\left(\frac{\partial \ln \rho}{\partial \ln \mathrm{P}}\right)_{\mu, T}$ and for an ideal gas $\alpha=\delta=\phi=1$, so that using pressure equilibrium $(\mathrm{dP}=0)$ we obtain $\Delta \ln \rho=\Delta \ln T$. In the case of a mixture of perfect gas and radiation we can write

$$
\Delta \ln \rho=-Q \Delta \ln T
$$

where

$$
Q=\frac{4-3 \beta}{\beta}-\left(\frac{\partial \ln \mu}{\partial \ln T}\right)_{\mathrm{P}},
$$

with $\beta=\mathrm{P}_{\text {Gas }} / \mathrm{P}$. Plugging Eq. 17 in 13 one obtains

$$
\bar{v}^{2}=\frac{1}{8} g Q(\Delta T(\lambda) / T) \lambda .
$$

and substituting the temperature excess as calculated in 4

$$
\bar{v}^{2}=\frac{1}{8} g Q \frac{\lambda^{2}}{H_{\mathrm{P}}}\left(\nabla-\nabla^{\prime}\right)
$$

so that the average convective velocity can be calculated

$$
\bar{v}=\frac{1}{2 \sqrt{2}} \lambda\left(\frac{g Q}{H_{\mathrm{P}}}\right)^{1 / 2}\left(\nabla-\nabla^{\prime}\right)^{1 / 2}=\frac{1}{2 \sqrt{2}} g \lambda\left(\frac{\rho Q}{\mathrm{P}}\right)^{1 / 2}\left(\nabla-\nabla^{\prime}\right)^{1 / 2} .
$$

Mixing length convective velocities depend linearly on the choice of the mixing length parameter $\lambda$. Also they depend on $Q^{1 / 2}$, which diverges for $\beta \rightarrow 0$, that is in radiation dominated regions. In these regions MLT often results in supersonic convective velocities. Note that the above calculations hold only in the case of pressure equilibrium, which is only justified if $\bar{v}<c_{s}$, i.e. for subsonic convection.

We can now rewrite the convective flux using the derived form of the convective velocities and Eq. 8

$$
F_{c}=\frac{1}{2} \rho \bar{v} c_{\mathrm{P}} T \frac{\lambda}{H_{\mathrm{P}}}\left(\nabla-\nabla^{\prime}\right)=\frac{1}{4 \sqrt{2}} g^{2} \rho^{5 / 2} c_{\mathrm{P}} Q^{1 / 2} T \mathrm{P}^{-3 / 2} \lambda^{2}\left(\nabla-\nabla^{\prime}\right)^{3 / 2} .
$$




\subsection{Convective Efficiency}

Energy losses (and gains) need to be accounted for during the convective element rise/fall. When these are not negligible, $\nabla \neq \nabla \neq \nabla_{\mathrm{ad}}$. In the deep interiors of stars energy loss can be due to neutrinos, while gains are produced by nuclear burning. In the outer layers however radiation is usually responsible for departure from adiabaticity. These gains/losses are regarded as purely "horizontal" in the sense that one has to think as the average effect created by all the convective elements over a shell. Therefore no direct contribution to the net vertical heat transport is expected (on average there are as many hot and cool elements crossing the shell), but an indirect contribution can arise due to the modification to the convective properties (e.g. convective velocities). Focusing on horizontal radiative losses, the usual assumption is that the convective element is optically thick. The change in temperature of a rising convective element is due to both its adiabatic expansion and the horizontal radiative losses. For optically thick turbulence, from the equation of radiative transfer (5)

$$
F=-\frac{4 a c}{3} \frac{T^{3}}{\kappa \rho} \frac{\Delta T}{\lambda / 2}
$$

with $\Delta T$ the temperature difference between the center of the turbulent element and the surrounding matter averaged over its lifetime. Given the area $A$ of the element and its lifetime $\lambda / \bar{v}$ the total radiated energy is

$$
E_{\text {rad }}=\frac{4 a c}{3} \frac{T^{3}}{\kappa \rho} \frac{\Delta T}{\lambda / 2} \frac{\lambda A}{\bar{v}} .
$$

The energy excess content carried by the convective element before dissolving is $c_{\mathrm{P}} \rho \Delta T^{*} V$ with $\Delta T^{*}$ here being the temperature excess of the element over its surroundings at the end of its path. Obviously $\Delta T^{*} \simeq$ $\Delta T$, and in the classic Böhm-Vitense work the choice $\Delta T^{*}=2 \Delta T$ is made. Using these quantities one can define a convective efficiency $\Gamma$ as

$$
\Gamma=\frac{\text { EnergyTransported }}{\text { EnergyLost }}=\frac{2 c_{\mathrm{P}} \rho \Delta T V}{\frac{4 a c}{3} \frac{T^{3}}{\kappa \rho} \frac{\Delta T}{\lambda / 2} \frac{\lambda A}{\bar{v}}}=\frac{c_{\mathrm{P}}}{6 a c} \frac{\kappa \rho^{2} \bar{v} \lambda}{T^{3}} \sim \frac{\bar{v} \lambda}{K} \equiv \mathrm{Pe}
$$

where we used the fact that $V / A=\lambda / 6$ for a sphere of diameter $\lambda$. Note how $\Gamma$ is related to the Peclet number Pe, which is defined as the ratio between the thermal and the dynamical timescales. The next step is substituting the calculated average velocity from 1.4 to obtain

$$
\Gamma=\frac{c_{\mathrm{P}}}{12 \sqrt{2} a c} \frac{\kappa g Q^{1 / 2} \rho^{5 / 2} \lambda^{2}}{\mathrm{P}^{1 / 2} T^{3}}\left(\nabla-\nabla^{\prime}\right)^{1 / 2}=A\left(\nabla-\nabla^{\prime}\right)^{1 / 2},
$$

where

$$
A \equiv \frac{Q^{1 / 2} c_{\mathrm{P}} \kappa g \rho^{5 / 2} \lambda^{2}}{12 \sqrt{2} a c \mathrm{P}^{1 / 2} T^{3}}
$$

is essentially the ratio of the convective to the radiative conductivities.

\subsection{Solutions for non-adiabatic convection}

The three basic equations to be solved involve three unknowns: $\nabla, \nabla^{\prime}$ and the convective efficiency $\Gamma$ :

$$
\Gamma=A\left(\nabla-\nabla^{\prime}\right)^{1 / 2} \nabla_{r}-\nabla=a_{0} A\left(\nabla-\nabla^{\prime}\right)^{3 / 2} \Gamma=\left(\nabla-\nabla^{\prime}\right) /\left(\nabla^{\prime}-\nabla_{\mathrm{ad}}\right)
$$

Here the numerical factor $a_{0}$ is of order 1 and differs slightly depending on different implementation of the MLT. These three equation can be rearranged to obtain

$$
\frac{\nabla_{r}-\nabla}{\nabla_{r}-\nabla_{\mathrm{ad}}} \equiv \zeta=\frac{a_{0} \Gamma^{2}}{1+\Gamma\left(1+a_{0} \Gamma\right)} .
$$


$\zeta$ is a function of the convective efficicency only, with $\zeta \rightarrow 0$ as $\Gamma \rightarrow 0$ and $\zeta \rightarrow 1$ as $\Gamma \rightarrow \infty$. It is possible to write a single cubic equation with $\zeta$, the new measure of convective efficicency, as the unknown variable. Once its value its determined the value of the actual gradient is given by $\nabla=(1-\zeta) \nabla_{r}+\zeta \nabla_{\text {ad }}$. Note that $\nabla \rightarrow \nabla_{r}$ for $\zeta \rightarrow 0$ and $\nabla \rightarrow \nabla_{\text {ad }}$ for $\zeta \rightarrow 1$ as expected since $\zeta$ is a measure of the convective efficiency. We can write

$$
\Gamma=B \zeta^{1 / 3},
$$

where $B \equiv\left[\left(A^{2} / a_{0}\right)\left(\nabla_{r}-\nabla_{\mathrm{ad}}\right)\right]^{1 / 3}$. With these definitions we finally obtain the cubic equation usually solved in MLT non-adiabatic convection:

$$
\zeta^{1 / 3}+B \zeta^{2 / 3}+a_{0} B^{2} \zeta-a_{0} B^{2}=0 .
$$

Once the value of $\zeta$ is known one can calculate the convective efficiency from 28, the value of the actual gradient from $\nabla=(1-\zeta) \nabla_{r}+\zeta \nabla_{\mathrm{ad}}$. To calculate the ratio of convective to total flux one can use 6 and write

$$
\frac{F_{c}}{F}=\frac{F-F_{r}}{F}=\frac{\nabla_{r}-\nabla}{\nabla_{r}}=\frac{\nabla_{r}-\nabla_{\mathrm{ad}}}{\nabla_{r}} \zeta .
$$

The convective velocities can be calculated obtaining $\nabla-\nabla^{\prime}$ from first eq. in 26 and subsituting the value in 1.4. Finally the superadiabaticity $\nabla-\nabla_{\mathrm{ad}}$ is obtained from $\nabla=(1-\zeta) \nabla_{r}+\zeta \nabla_{\mathrm{ad}}$, which gives:

$$
\nabla-\nabla_{\mathrm{ad}}=(1-\zeta)\left(\nabla_{r}-\nabla_{\mathrm{ad}}\right) .
$$

